\title{
SOME REMARKS ABOUT PARAMETRIZATIONS OF INTRINSIC REGULAR SURFACES IN THE HEISENBERG GROUP
}

\author{
Francesco Bigolin and Davide Vittone
}

\begin{abstract}
We prove that, in general, $\mathbb{H}$-regular surfaces in the Heisenberg group $\mathbb{H}^{1}$ are not bi-Lipschitz equivalent to the plane $\mathbb{R}^{2}$ endowed with the "parabolic" distance, which instead is the model space for $C^{1}$ surfaces without characteristic points. In Heisenberg groups $\mathbb{H}^{n}, \mathbb{H}$-regular surfaces can be seen as intrinsic graphs: we show that such parametrizations do not belong to Sobolev classes of metric-space valued maps.
\end{abstract}

\section{Introduction}

In the last few years, a systematic attempt to develop Analysis and Geometry in Metric Spaces has become the object of many studies. In particular, the Heisenberg group $\mathbb{H}^{n}$, endowed with the so called CarnotCarathéodory distance $d_{c}$, has provided a fruitful setting for these investigations. One of the main questions which have arisen concerns how to develop a general theory of "intrinsically regular" hypersurfaces: in [10] B. Franchi, R. Serapioni and F. Serra Cassano introduced the notion of $\mathbb{H}$-regular hypersurface, which was later proved to be a "good" one by several evidences provided by different authors. It is easy to see that Euclidean $C^{1}$ surfaces without characteristic points (see [4]) are in fact $\mathbb{H}$-regular. On the contrary, there is a huge gap between these two notions, since $\mathbb{H}$-regular surfaces can be fractals from the Euclidean viewpoint (see [12]). Accurate studies of $\mathbb{H}$-regular surfaces have been

2000 Mathematics Subject Classification. 53C17, 54E40.

Key words. Bi-Lipschitz parametrizations, $\mathbb{H}$-regular surfaces, Heisenberg group. F. B. was partially supported by GALA project "Geometric Analysis in Lie groups and Applications" of the European Commission, by PRIN, MIUR, Italy, GNAMPA of INDAM and University of Trento, Italy.

D. V. was partially supported by GALA project "Geometric Analysis in Lie groups and Applications" of the European Commission, by PRIN, MIUR, Italy, GNAMPA of INDAM and University of Padova, Italy. 
carried out in $[\mathbf{2}],[\mathbf{5}],[\mathbf{7}]$; we address the reader to $[\mathbf{6}],[\mathbf{1 5}]$ for a more comprehensive introduction to the Heisenberg group and $\mathbb{H}-$-regular hypersurfaces.

A problem raised in [10], directly related to the theory of rectifiability in the Heisenberg group (see also [1], [10], [11], [13], [14]), is the following one: is it possible to see $\mathbb{H}$-regular hypersurfaces as bi-Lipschitz deformations of a given "model" metric space? Here, by bi-Lipschitz we mean Lipschitz continuous maps with Lipschitz continuous inverse map. In [8] D. R. Cole and S. D. Pauls have proved that, in the setting of the first Heisenberg group $\mathbb{H}^{1}$, any noncharacteristic $C^{1}$ surface $S$ can be locally parametrized by means of a Lipschitz homeomorphism defined on (an open subset of) the plane $\mathbb{R}_{\eta, \tau}^{2}$ endowed with the "parabolic" distance

$$
\varrho\left((\eta, \tau),\left(\eta^{\prime}, \tau^{\prime}\right)\right):=\left|\eta-\eta^{\prime}\right|+\left|\tau-\tau^{\prime}\right|^{1 / 2} .
$$

Cole-Pauls homeomorphism is indeed bi-Lipschitz continuous: see Theorem 3.1. The question of understanding whether general $\mathbb{H}$-regular surfaces are bi-Lipschitz equivalent to the parabolic plane was left open. We give a negative answer to this problem, thus providing a further evidence of the gap between $C^{1}$ and genuine $\mathbb{H}$-regular surfaces. Our main result is in fact the following

Theorem 1.1. There exists a $\mathbb{H}$-regular hypersurface $S \subset \mathbb{H}^{1}$ and $a$ point $P \in S$ with the following property: any Lipschitz continuous map $\Psi: \mathcal{A} \rightarrow \mathcal{U}$ from an open set $\mathcal{A} \subset \mathbb{R}^{2}$ to a neighbourhood $\mathcal{U}$ of $P$ on $S$ cannot be a homeomorphism. In particular, there cannot exist a biLipschitz parametrization $\Psi:(\mathcal{A}, \varrho) \rightarrow\left(\mathcal{U}, d_{c}\right)$.

In the paper [10] an Implicit Function Theorem for $\mathbb{H}$-regular surfaces was proved, showing that such a surface $S \subset \mathbb{H}^{n}$ can be locally parametrized as the intrinsic graph $\Phi: \omega \rightarrow S$ of a function $\phi: \omega \rightarrow \mathbb{R}$. Here $\omega$ is an open subset of a maximal subgroup $\mathbb{W}$ of $\mathbb{H}^{n}$. It was found in [2] that such $\Phi$ is bi-Lipschitz continuous provided we endow $\omega$ with a certain quasi-distance $\rho_{\phi}$ depending on $\phi$ itself. It would be interesting to investigate the regularity of $\Phi:(\omega, d) \rightarrow\left(S, d_{c}\right)$ with respect to some "fixed" distance $d$ on $\omega$; in this sense, a question risen in [12] was to understand whether the map $\Phi$ belongs to some Sobolev class $W_{m}^{1, p}\left((\omega, d),\left(\mathbb{H}^{n}, d_{c}\right)\right)$ of maps between metric spaces. At least in the $\mathbb{H}^{1}$ case (see Remark 4.3 therein) it was conjectured that the parametrization $\Phi$ should belong to $W_{m}^{1,4}\left((\omega, d),\left(\mathbb{H}^{n}, d_{c}\right)\right)$, where the distance on $\omega$ is the Euclidean one on $\mathbb{W} \equiv \mathbb{R}^{2}$. We are able to answer in the negative also to this second question: 
Theorem 1.2. The intrinsic parametrization $\Phi: \omega \rightarrow S$ of a $\mathbb{H}$-regular surface $S$ does not belong to any Sobolev space $W_{m}^{1, p}\left((\omega, d),\left(\mathbb{H}^{n}, d_{c}\right)\right)$ for $1 \leq p \leq+\infty$ when $d$ is the Euclidean distance on $\omega$. The same result holds when $d$ is the distance $d_{c}$ on $\omega \subset \mathbb{H}^{n}$ provided $\Phi$ is not the inclusion map $\omega \hookrightarrow \mathbb{H}^{n}$ (i.e. if $\left.\phi \not \equiv 0\right)$.

The paper is organized as follows. Section 2 is devoted to the presentation of the preliminary material. In Section 3 we give the proof of Theorem 1.1, while Section 4 contains the one of Theorem 1.2.

Acknowledgements. We wish to express our gratitude to Zoltán M. Balogh and Giovanna Citti, for having signaled us the problem of the biLipschitz parametrization of $\mathbb{H}$-regular surfaces and for many invaluable discussions. We have also to thank Francesco Serra Cassano for several suggestions.

\section{Notations and preliminary results}

We will identify $\mathbb{H}^{n}$ with $\mathbb{R}^{2 n+1}$ by means of the coordinates

$$
\mathbb{H}^{n} \ni P \longleftrightarrow(x, y, t) \in \mathbb{R}^{n} \times \mathbb{R}^{n} \times \mathbb{R}
$$

according to which the group law reads as

$$
(x, y, t) \cdot\left(x^{\prime}, y^{\prime}, t^{\prime}\right)=\left(x+x^{\prime}, y+y^{\prime}, t+t^{\prime}+\frac{1}{2} \sum_{j=1}^{n}\left(x_{j} y_{j}^{\prime}-y_{j} x_{j}^{\prime}\right)\right) .
$$

The group identity is 0 and the inverse element $(x, y, t)^{-1}$ is $(-x,-y,-t)$. The Lie algebra of left invariant vector fields is generated by

$$
X_{j}=\partial_{x_{j}}-\frac{y_{j}}{2} \partial_{t}, \quad Y_{j}=\partial_{y_{j}}+\frac{x_{j}}{2} \partial_{t}, \quad \text { for } j=1, \ldots, n ; \quad T=\partial_{t},
$$

where as usual we identified vector fields and first order differential operators. The only nonvanishing commutation relationships are given by $\left[X_{j}, Y_{j}\right]=T$ for any $j=1, \ldots, n$. For computational convenience, instead of the usual Carnot-Carathéodory distance $d_{c}$ we will consider the equivalent distance $d_{\infty}$ on $\mathbb{H}^{n}$ arising from the homogeneous norm

$$
\|P\|_{\infty}:=\max \left\{|(x, y)|,|t|^{1 / 2}\right\}
$$

i.e. $d_{\infty}(P, Q):=\left\|Q^{-1} \cdot P\right\|_{\infty}$. Absolutely continuous curves $\gamma$ with $\gamma^{\prime}(s) \in \operatorname{span}\left\{X_{1}, \ldots, Y_{n}\right\}$ for a.e. $s \in \mathbb{R}$ are called horizontal.

A continuous function $f: \Omega \subset \mathbb{H}^{n} \rightarrow \mathbb{R}$ is of class $C_{\mathbb{H}}^{1}(\Omega)$ if its horizontal derivatives

$$
\nabla_{\mathbb{H}} f:=\left(X_{1} f, \ldots, X_{n} f, Y_{1} f, \ldots, Y_{n} f\right)
$$

are represented, in distributional sense, by continuous functions on the open set $\Omega$. The inclusion $C^{1}(\Omega) \subset C_{\mathbb{H}}^{1}(\Omega)$ is strict; see for example $[\mathbf{1 0}$, Remark 5.9]. 
Definition 2.1. We shall say that $S \subset \mathbb{H}^{n}$ is a $\mathbb{H}$-regular hypersurface if for every $P \in S$ there exist an open ball $B(P, r)$ and a function $f \in$ $\mathbf{C}_{\mathbb{H}}^{1}(B(P, r))$ such that

$S \cap B(P, r)=\{Q \in B(P, r): f(Q)=0\} \quad$ and $\quad \nabla_{\mathbb{H}} f \neq 0$ on $B(P, r)$.

Euclidean $C^{1}$ surfaces are $\mathbb{H}$-regular provided they have no characteristic points, i.e. no points where the tangent plane coincides with $\operatorname{span}\left\{X_{1}, \ldots, Y_{n}\right\}$.

It will not be restrictive to consider only $\mathbb{H}$-regular surfaces given as level sets $\{f=0\}$ of $C_{\mathbb{H}}^{1}$ functions with $X_{1} f>0$. With this assumption in mind, in the following we fix $\mathbb{W}$ to be the maximal subgroup of $\mathbb{H}^{n}$ defined by

$\mathbb{W}:=\exp \left(\operatorname{span}\left\{X_{2}, \ldots, X_{n}, Y_{1}, \ldots, Y_{n}, T\right\}\right)=\left\{(x, y, t) \in \mathbb{R}^{2 n+1}: x_{1}=0\right\}$.

We also write $(y, t)$ to denote a point $(0, y, t) \in \mathbb{W}$ if $n=1$; if $n \geq 2$ we write $\left(y_{1}, z, t\right) \in \mathbb{R} \times \mathbb{R}^{2 n-2} \times \mathbb{R}$ instead of $\left(0, x_{2}, \ldots, x_{n}, y_{1}, \ldots, y_{n}, t\right) \in$ $\mathbb{W}$, where $z=\left(x_{2}, \ldots, x_{n}, y_{2}, \ldots, y_{n}\right)$.

Theorem 2.2 (Implicit Function Theorem, [10]). Let $S=\{f=0\}$ be a $\mathbb{H}$-regular hypersurface given as the level set of a $C_{\mathbb{H}}^{1}$ function $f$ with $X_{1} f>0$. Then, locally on $S$, there exists a unique continuous map $\phi: \omega \subset \mathbb{W} \rightarrow \mathbb{R}$ such that $S=\Phi(\omega)$, where $\Phi$ is defined by

$$
\Phi(A):=A \cdot(\phi(A), 0, \ldots, 0)=\exp \left(\phi(A) X_{1}\right)(A) .
$$

Moreover, $\Phi$ is a homeomorphism.

The explicit structure of $\Phi$ is given by

$$
\begin{array}{rr}
\Phi(y, t)=\left(\phi(y, t), y, t-\frac{1}{2} y \phi(y, t)\right), & \text { if } n=1 \\
\Phi\left(y_{1}, z, t\right)=\left(\phi\left(y_{1}, z, t\right), x_{2}, \ldots, x_{n}, y_{1}, y_{2}, \ldots, y_{n},\right. & \\
\left.t-\frac{1}{2} y_{1} \phi\left(y_{1}, z, t\right)\right), & \text { if } n \geq 2 .
\end{array}
$$

This not being restrictive, we will always suppose that the whole surface $S$ coincides with the image of $\Phi$. It was proved in [12] that $\Phi$ is $1 / 2$-Hölder continuous (in fact, also slightly better, see Corollary 4.5 in $[\mathbf{2}])$ when the distance on $\omega \subset \mathbb{W} \equiv \mathbb{R}^{2 n}$ is the Euclidean one, but not, in general, of class $C^{0, \alpha}$ for $\alpha>\frac{1}{2}$.

The problem of the regularity of $\phi$ and $\Phi$, that from now on will always be related by (2.1), was addressed in $[\mathbf{2}],[\mathbf{5}],[\mathbf{7}]$. In particular, in [2] it was proved that the map $\Phi: \omega \rightarrow S$ is bi-Lipschitz continuous provided we endow $\omega$ with a certain quasi-distance $\rho_{\phi}$, that depends on $\phi$ itself. Explicitly, for $A, B \in \omega$ one has 
- if $n=1, A=(y, t), B=\left(y^{\prime}, t^{\prime}\right)$, then $\rho_{\phi}(A, B):=\left|y^{\prime}-y\right|+\left|t^{\prime}-t-\frac{1}{2}(\phi(A)+\phi(B))\left(y^{\prime}-y\right)\right|^{1 / 2} ;$

- if $n \geq 2, A=\left(y_{1},\left(x_{2}, \ldots, x_{n}, y_{2}, \ldots, y_{n}\right), t\right), B=\left(y_{1}^{\prime},\left(x_{2}^{\prime}, \ldots, x_{n}^{\prime}\right.\right.$, $\left.\left.y_{2}^{\prime}, \ldots, y_{n}^{\prime}\right), t\right)$, then

$$
\begin{aligned}
& \rho_{\phi}(A, B):=\sum_{j=2}^{n}\left|x_{j}^{\prime}-x_{j}\right|+\sum_{j=1}^{n}\left|y_{j}^{\prime}-y_{j}\right| \\
& \quad+\left|t^{\prime}-t-\frac{1}{2}(\phi(A)+\phi(B))\left(y_{1}^{\prime}-y_{1}\right)+\frac{1}{2} \sum_{j=2}^{n}\left(x_{j}^{\prime} y_{j}-x_{j} y_{j}^{\prime}\right)\right|^{1 / 2} .
\end{aligned}
$$

If $S$ is $\mathbb{H}$-regular, $\rho_{\phi}$ turns out to be a quasi-metric, i.e. the triangular inequality is replaced by

$$
\rho_{\phi}(A, B) \leq K\left(\rho_{\phi}(A, C)+\rho_{\phi}(C, B)\right) \quad \text { for any } A, B, C \in \omega
$$

for a certain $K \geq 1$. The problem of finding a bi-Lipschitz parametrization of $S$ is therefore equivalent to that of finding a bi-Lipschitz parametrization of $\left(\omega, \rho_{\phi}\right)$. Moreover, curves on $S$ with finite length (i.e. horizontal curves) correspond, via $\Phi$, to curves with finite length in $\left(\omega, \rho_{\phi}\right)$.

It turns out that the parametrization $\phi$ of a $\mathbb{H}$-regular surface $S$ is "regular" (i.e. differentiable in a specific sense, see [2], [7]) along the directions

$$
\begin{array}{ll}
X_{j}=\partial_{x_{j}}-\frac{y_{j}}{2} \partial_{t} & \text { for } 2 \leq j \leq n \\
W^{\phi}:=Y_{1}+\phi T=\partial_{y_{1}}+\phi \partial_{t} & \\
Y_{j}=\partial_{y_{j}}+\frac{x_{j}}{2} \partial_{t} & \text { for } 2 \leq j \leq n .
\end{array}
$$

Remark 2.3. We will not enter into details regarding the intrinsic gradient $\nabla^{\phi} \phi:=\left(X_{1} \phi, \ldots, X_{n} \phi, W^{\phi} \phi, Y_{2} \phi, \ldots, Y_{n} \phi\right)$ of parametrizations $\phi$ of $\mathbb{H}$-regular surfaces: we refer again to [2], [7]. Let us only recall that $\nabla^{\phi} \phi: \omega \rightarrow \mathbb{R}^{2 n-1}$ is a continuous function which determines the horizontal normal to the surface.

$$
\nu_{S}(P)=\left(-\frac{1}{\sqrt{1+\left|\nabla^{\phi} \phi\right|^{2}}}, \frac{\nabla^{\phi} \phi}{\sqrt{1+\left|\nabla^{\phi} \phi\right|^{2}}}\right)\left(\Phi^{-1}(P)\right) .
$$

In particular, horizontal curves on $S$ have derivatives lying in the linear space generated by the vectors

$$
\begin{array}{ll}
\left(\left(X_{j} \phi\right) \circ \Phi^{-1}\right) X_{1}+X_{j} & (j=2, \ldots, n) \\
\left(\left(W^{\phi} \phi\right) \circ \Phi^{-1}\right) X_{1}+Y_{1} & \\
\left(\left(Y_{j} \phi\right) \circ \Phi^{-1}\right) X_{1}+Y_{j} & (j=2, \ldots, n) .
\end{array}
$$


In fact, the derivative of such a curve at a point $P$, whenever it exists, belongs to the blow up of $S$ at $P$, which is the "vertical" maximal subgroup "orthogonal" to $\nu_{S}(P)$.

In the sequel we will need the following result, which allows to exhibit examples of $\mathbb{H}$-regular surfaces in $\mathbb{H}^{1}$ which are not of class $C^{1}$.

Theorem 2.4 ([2, Corollary 5.11]). Suppose $n=1$ and $\phi: \omega \subset \mathbb{W} \equiv$ $\mathbb{R}_{y, t}^{2} \rightarrow \mathbb{R}$ is a continuous map depending only on the second variable $t$, namely $\phi(y, t)=\varphi(t)$. Assume that $\varphi^{2}$ is of class $C^{1}$; then the image $\Phi(\omega)$ is a $\mathbb{H}$-regular surface in $\mathbb{H}^{1}$. Moreover, $\left(W^{\phi} \phi\right)(y, t)=$ $\frac{1}{2}\left(\varphi^{2}\right)^{\prime}(t)$.

Finally, we need to define Sobolev classes of maps between metric spaces; we refer to $[\mathbf{3}]$ and the references therein. Given two metric spaces $\left(M, d_{M}\right),\left(N, d_{N}\right)$, a nonnegative Radon measure $\mu$ on $M$ and $1 \leq p \leq+\infty$, we will say that $u: M \rightarrow N$ belongs to the Sobolev space $\overline{W_{m}^{1}, p}\left(\left(M, d_{M}\right),\left(N, d_{N}\right)\right)$ if there exists $g: M \rightarrow[0,+\infty]$ with $g \in$ $L^{p}(M, d \mu)$ and

(2.4) $d_{N}(u(A), u(B)) \leq(g(A)+g(B)) d_{M}(A, B) \quad$ for any $A, B \in M$.

We will always be concerned with the case $M=\omega,\left(N, d_{N}\right)=\left(\mathbb{H}^{n}, d_{\infty}\right)$ and $\mu=\mathcal{L}^{2 n}$.

\section{Proof of Theorem 1.1}

As mentioned in the Introduction, $\mathbb{H}$-regular surfaces of class $C^{1}$ can be modeled on the parabolic plane (1.1), as specified in the following result. The first part of the statement was proved by D. R. Cole and S. D. Pauls [8].

Theorem 3.1. Let $S$ be a $C^{1}$ surface; then for any non characteristic point $P \in S$ there is a Lipschitz continuous homeomorphism

$$
\Psi:(\mathcal{A}, \varrho) \longrightarrow\left(\mathcal{U}, d_{\infty}\right),
$$

from an open set $\mathcal{A} \subset \mathbb{R}^{2}$ to a neighbourhood $\mathcal{U}$ of $P$ in $S$, with Lipschitz continuous inverse map $\Psi^{-1}$.

Proof: It is not restrictive to suppose that $P=0$ and that a neighbourhood $\mathcal{U} \subset S$ of 0 is parametrized by a $C^{1}$ function $\phi: \omega \rightarrow \mathbb{R}$ with $\phi(0)=0$. Let us introduce the map

$$
\begin{aligned}
\psi: \quad \mathcal{A} & \rightarrow \omega \\
(\eta, \tau) & \mapsto \exp \left(\eta W^{\phi}\right)(0, \tau)
\end{aligned}
$$


which is defined, possibly restricting $\omega$, on a proper open set $\mathcal{A} \subset \mathbb{R}^{2}$. It is not difficult to notice that the Lipschitz homeomorphism $\Psi: \mathcal{A} \rightarrow \mathcal{U}$ introduced by D. R. Cole and S. D. Pauls is $\Psi=\Phi \circ \psi$. Since $\Phi$ is a $\left(\rho_{\phi^{-}} d_{\infty}\right)$ bi-Lipschitz homeomorphism, it will be sufficient to show that the inverse map $\psi^{-1}$ is $\left(\rho_{\phi^{-}} \varrho\right)$-Lipschitz continuous.

To this aim, for any $A=(y, t) \in \omega$ let us introduce the curve $\tau_{A}$ solution of the ODE

$$
\tau_{A}(y)=t, \quad \dot{\tau}_{A}(s)=\phi\left(s, \tau_{A}(s)\right) .
$$

It is immediate to see that $\psi^{-1}(A)=\psi^{-1}(y, t)=\left(y, \tau_{A}(0)\right)$. The Lipschitz estimate we need to prove is therefore

$\left|y^{\prime}-y\right|+\left|\tau_{B}(0)-\tau_{A}(0)\right|^{1 / 2} \leq c \rho_{\phi}(A, B) \quad \forall A=(y, t), B=\left(y^{\prime}, t^{\prime}\right) \in \omega$. If $y^{\prime}=y$ we have

$$
\begin{aligned}
\left|\tau_{B}(0)-\tau_{A}(0)\right|=\mid t^{\prime}-t+\int_{y}^{0}[ & \left.\phi\left(s, \tau_{B}(s)\right)-\phi\left(s, \tau_{A}(s)\right)\right] d s \mid \\
& \leq\left|t^{\prime}-t\right|+c_{1} \int_{y}^{0}\left|\tau_{B}(s)-\tau_{A}(s)\right| d s
\end{aligned}
$$

and by Gronwall's lemma one concludes that

$$
\left|\tau_{B}(0)-\tau_{A}(0)\right|^{1 / 2} \leq c_{2}\left|t^{\prime}-t\right|^{1 / 2}=\rho_{\phi}(A, B) .
$$

If $y^{\prime} \neq y$ we define $C:=\exp \left(\left(y-y^{\prime}\right) W^{\phi}\right)(B)=\left(y, t^{\prime \prime}\right)$. We refer to $[\mathbf{2}$, Theorem 3.8] for the proof of the inequality $\left|t^{\prime \prime}-t^{\prime}\right|^{1 / 2} \leq c_{3} \rho_{\phi}(A, B)$; with this in our hands we can conclude in a stroke since

$$
\begin{aligned}
\left|y^{\prime}-y\right|+\left|\tau_{B}(0)-\tau_{A}(0)\right|^{1 / 2} & =\left|y^{\prime}-y\right|+\left|\tau_{C}(0)-\tau_{A}(0)\right|^{1 / 2} \\
& \leq\left|y^{\prime}-y\right|+c_{2}\left|t^{\prime \prime}-t\right|^{1 / 2} \\
& \leq c \rho_{\phi}(A, B) .
\end{aligned}
$$

As we said, Theorem 3.1 fails to hold for general $\mathbb{H}$-regular surfaces: the idea for constructing a counterexample lies in the possibility of finding $\mathbb{H}$-regular surfaces which are connected by curves with finite length, and to notice that the parabolic plane does not share this property. Clearly, $C^{1}$ noncharacterictic surfaces are not connected by means of such curves.

Let us consider the intrinsic graph $S:=\Phi(\mathbb{W})$ of the map

$$
\phi(y, t):= \begin{cases}-\frac{t^{\alpha}}{1-\alpha} & \text { if } t \geq 0 \\ 0 & \text { if } t<0 .\end{cases}
$$


The exponent $\alpha$ is such that $\frac{1}{2}<\alpha<1: S$ is therefore $\mathbb{H}$-regular thanks to Theorem 2.4 and $\left(W^{\phi} \phi\right)(y, t)=\frac{\alpha}{(1-\alpha)^{2}} t^{2 \alpha-1}$ if $t \geq 0,\left(W^{\phi} \phi\right)(y, t)=0$ otherwise. The surface is constituted by the union of the two $C^{1}$ surfaces

$$
\begin{aligned}
& S_{+}:=\left\{(x, y, t) \in \mathbb{H}^{n}: t+\frac{1}{2} x y>0, x=-\frac{1}{1-\alpha}\left(t+\frac{1}{2} x y\right)^{\alpha}\right\} \\
& S_{-}:=\left\{(0, y, t) \in \mathbb{H}^{1}: t<0\right\}
\end{aligned}
$$

glued together along the horizontal line $L:=\{(0, y, 0): y \in \mathbb{R}\} ; S$ is clearly not $C^{1}$ regular at points of $L$.

Proof of Theorem 1.1: Let us consider the $\mathbb{H}$-regular surface $S$ parametrized by the map $\phi$ in $(3.1)$ and the point $P=(0,0,0) \in S$; let $\Psi$ be as in the statement of the theorem.

For any fixed $\tau$ the curve $\gamma_{\tau}:=\Psi(\cdot, \tau): \mathbb{R} \rightarrow \mathbb{H}^{1}$ is Lipschitz continuous; therefore (see Remark 2.3) it is tangent to the vector field $W:=Y_{1}+\left(W^{\phi} \phi \circ \Phi^{-1}\right) X_{1}$ on $S$. In particular, the support of $\gamma_{\tau}$ is contained in integral lines of $W$. It is a matter of fact that the projection $\Phi^{-1}(\gamma)$ of an integral curve $\gamma$ of $W$ is an integral curve of $W^{\phi}$ in $\mathbb{W}$.

Let us investigate the behaviour of the integral curves of $W^{\phi}$, i.e. the solutions of the Cauchy problem

$$
c^{\prime}(s)=W^{\phi}(c(s))=\partial_{y}+\phi(c(s)) \partial_{t} .
$$

More precisely, if $c(s)=\left(c_{y}(s), c_{t}(s)\right)$ we have

$$
c_{y}^{\prime}=1 \quad \text { and } \quad c_{t}^{\prime}=-\frac{c_{t}^{\alpha}}{1-\alpha} .
$$

Lipschitz regularity of the coefficients of this ODE is violated at points $(y, 0)$, therefore we cannot expect uniqueness of solutions whenever $c_{t}=0$. By standard considerations on this kind of problem we can divide the solutions of the ODE into two families $\left\{c_{w}^{+}\right\}_{w \in \mathbb{R}}$ and $\left\{c_{\zeta}^{-}\right\}_{\zeta \leq 0}$ :

$$
\begin{aligned}
& c_{w}^{+}(s)= \begin{cases}\left(s,(w-s)^{\frac{1}{1-\alpha}}\right) & \text { if } s \leq w \\
(s, 0) & \text { if } s \geq w\end{cases} \\
& c_{\zeta}^{-}(s)=(s, \zeta) .
\end{aligned}
$$

Notice that, for a given curve $c_{w}^{+}$, the parameter $w$ denotes the point $(w, 0)$ where it meets the horizontal axis $y$. We will also write $c_{w}^{++}$to denote the restriction of $c_{w}^{+}$to $\left.]-\infty, w\right]$, i.e. the part of $c_{w}^{+}$lying in the upper halfplane. The upper (closed) halfplane is connected by means of $c_{0}^{-}$and of paths of type $c_{w}^{+}$. 
It will not be restrictive to suppose $\Psi(0,0)=0 \in S$ and $\mathcal{U}=\Phi(]-\delta, \delta\left[^{2}\right)$ for some positive $\delta$. Let us denote by $\psi$ the $\left(\varrho-\rho_{\phi}\right)$-Lipschitz induced map $\left.\psi:=\Phi^{-1} \circ \Psi: \mathcal{A} \rightarrow\right]-\delta, \delta\left[^{2}\right.$, which is such that $\psi(0,0)=(0,0)$; suppose by contradiction that it is also a homeomorphism. Then the set

$$
K:=\psi^{-1}\{(0, t): t \in[0, \delta / 2]\}
$$

is a compact subset of $\mathcal{A}$ and for sufficiently small $r>0$

$$
\{(\eta+h, \tau):(\eta, \tau) \in K,-r \leq h \leq r\} \subset \mathcal{A} .
$$

Let us set

$$
\begin{aligned}
& r_{+}:=\sup \{\eta>0: \psi(\eta, 0) \in \mathbb{R} \times\{0\}\} \geq 0 \\
& r_{-}:=\inf \{\eta<0: \psi(\eta, 0) \in \mathbb{R} \times\{0\} \leq 0 .
\end{aligned}
$$

One cannot have $r_{+}=r_{-}=0$; indeed, this would imply

$\{\psi(\eta, 0): \eta>0\} \subset \operatorname{Im} c_{0}^{++} \backslash\{0\}$ and $\{\psi(\eta, 0): \eta<0\} \subset \operatorname{Im} c_{0}^{++} \backslash\{0\}$, and by continuity $(\psi(0,0)=0)$ we obtain

$$
\{\psi(\eta, 0): \eta>0\} \cap\{\psi(\eta, 0): \eta<0\} \neq \emptyset
$$

i.e. $\psi$ is not injective, a contradiction.

Therefore, one between $r_{+}$and $r_{-}$is nonzero: by substituting $\psi$ with $\psi(-\eta, \tau)$ if necessary, we can suppose that $r_{+}>0$. One has

$$
\left\{\psi(\eta, 0): 0 \leq \eta \leq r_{+}\right\} \subset \mathbb{R} \times\{0\},
$$

otherwise the curve $\left.\psi(\cdot, 0)\right|_{\left[0, r_{+}\right]}$would "leave" the horizontal axis $\mathbb{R} \times$ $\{0\}$ and then "return" on it after some time. This could be possible only by covering forward and then backward a piece of some $c_{w}^{++}$, and contradicting in particular the injectivity of $\psi$. We can choose $r \in] 0, r_{+}[$ such that (3.4) holds. Set $A:=\psi(r, 0)=(\bar{y}, 0)$; by continuity one must have

$$
\begin{array}{ll}
{[0, \bar{y}] \times\{0\} \subset\{\psi(\eta, 0): 0 \leq \eta<r\}} & \text { if } \bar{y}>0 \\
{[\bar{y}, 0] \times\{0\} \subset\{\psi(\eta, 0): 0 \leq \eta<r\}} & \text { if } \bar{y}<0 .
\end{array}
$$

Since $A \neq 0$ (i.e. $\bar{y} \neq 0$ ) we easily find an $\epsilon>0$ such that

$$
\mathcal{V}_{1} \cap \mathcal{V}_{2}=\emptyset
$$

where

$$
\mathcal{V}_{1}:=\bigcup_{0<w<\epsilon} \operatorname{Im} c_{w}^{++} \quad \text { and } \quad \mathcal{V}_{2}:=\bigcup_{\bar{y}-\epsilon<w<\bar{y}+\epsilon} \operatorname{Im} c_{w}^{++}
$$


Notice that $A \in \mathcal{V}_{2}$, since $A \in c_{\bar{y}}^{++}$. Now, it is not difficult to prove that, in order to join a point $A_{1} \in \mathcal{V}_{1}$ with a point $A_{2} \in \mathcal{V}_{2}$ by following only integral lines of $W^{\phi}$, one must cover the whole segment $I$ defined by

$$
I:=[\epsilon, \bar{y}-\epsilon] \times\{0\} \text { if } \bar{y}>0, \quad I:=[\bar{y}+\epsilon, 0] \times\{0\} \text { if } \bar{y}<0 .
$$

Setting $\left(\eta_{t}, \tau_{t}\right):=\psi^{-1}(0, t)$, one has

$$
\lim _{t \rightarrow 0} \psi\left(\eta_{t}+r, \tau_{t}\right)=\psi(r, 0)=A .
$$

For sufficiently small $t>0$ the curve $\psi\left(\cdot, \tau_{t}\right)$ joins $A_{1}:=(0, t) \in \mathcal{V}_{1}$ to $A_{2}:=\psi\left(\eta_{t}+r, \tau_{t}\right)$ following only integral lines of $W^{\phi}$; moreover, $A_{2}$ must belong to $\mathcal{V}_{2}$. This implies that $I \subset \operatorname{Im} \psi\left(\cdot, \tau_{t}\right)$; since (see (3.5)) we have also $I \subset \operatorname{Im} \psi(\cdot, 0)$, this would contradict the injectivity of $\psi$ provided we are able to choose a sufficiently small $t$ such that $\tau_{t} \neq 0$. Were this not possible, there would exist $\lambda>0$ such that $\psi^{-1}(0, t)=\left(\eta_{t}, 0\right)$ for any $t \in[0, \lambda]$, i.e.

$$
\{0\} \times[0, \lambda] \subset \operatorname{Im} \psi(\cdot, 0) .
$$

Therefore the image $\Phi(\{0\} \times[0, \lambda])$ would be a horizontal curve, while it can be easily checked that this is not the case. A contradiction arises and the proof is completed.

Remark 3.2. In the spirit of Federer's approach to rectifiability see [9], [1]) it would be interesting to understand if $\mathbb{H}$-regular surfaces can be seen as Lipschitz images of the parabolic plane. In this sense, Theorem 1.1 essentially says that one cannot expect injectivity of the parametrization, since the images on $S$ of horizontal lines $(\cdot, t) \subset\left(\mathbb{R}^{2}, \varrho\right)$ are somehow "forced to meet".

The surface $S$ can be locally parametrized by means of Lipschitz images of the parabolic plane. This clearly follows from Theorem 3.1 for neighbourhoods of points in $S_{+} \cup S_{-}$. For points $P \in L$, it will be sufficient to observe that $P^{-1} S=S$ (thus reducing to the case $P=0$ ) and to show that the map

$$
\psi(\eta, \tau):= \begin{cases}c_{\tau-1}^{+}(\eta) & \text { if } \tau>0 \\ c_{\tau}^{-}(\eta) & \text { if } \tau \leq 0\end{cases}
$$

is $\left(\varrho-\rho_{\phi}\right)$-Lipschitz continuous from a neighbourhood of $(0,0)$ to a neighbourhood of $(0,0)$ in $\mathbb{W}$. Explicitly, we have

$$
\psi(\eta, \tau)= \begin{cases}\left(\eta,(\tau-1-\eta)^{1 / 1-\alpha}\right) & \text { if } \tau>0 \text { and } \eta \leq \tau-1 \\ (\eta, 0) & \text { if } \tau>0 \text { and } \eta \geq \tau-1 \\ (\eta, \tau) & \text { if } \tau \leq 0 .\end{cases}
$$

Clearly, $\psi$ is not injective, as $\psi(0, \tau)=(0,0)$ for any $\tau \in[0,1]$. 
It is not difficult (see [2]) to show that integral curves of $W^{\phi}$ are (locally) Lipschitz continuous with respect to $\rho_{\phi}$; in particular

$$
\rho_{\phi}\left(\psi\left(\eta_{1}, \tau\right), \psi\left(\eta_{2}, \tau\right)\right) \leq c\left|\eta_{1}-\eta_{2}\right|
$$

for $\left(\eta_{1}, \tau\right),\left(\eta_{2}, \tau\right)$ in a neighbourhood of $(0,0)$. It will therefore be sufficient to prove that

$$
\rho_{\phi}\left(\psi\left(\eta, \tau_{1}\right), \psi\left(\eta, \tau_{2}\right)\right) \leq C\left|\tau_{1}-\tau_{2}\right|^{1 / 2}
$$

for some $C>0$ and any $\left(\eta, \tau_{1}\right),\left(\eta, \tau_{2}\right)$ in a neighbourhood of $(0,0)$. We have several cases to take into account. If $\tau_{1}, \tau_{2}>0, \eta \leq \tau_{1}-1$ and $\eta \leq \tau_{2}-1$ then

$$
\begin{array}{r}
\rho_{\phi}\left(\psi\left(\eta, \tau_{1}\right), \psi\left(\eta, \tau_{2}\right)\right)=\left|\left(\tau_{1}-1-\eta\right)^{1 / 1-\alpha}-\left(\tau_{2}-1-\eta\right)^{1 / 1-\alpha}\right|^{1 / 2} \\
\leq C\left|\tau_{1}-\tau_{2}\right|^{1 / 2}
\end{array}
$$

where we used that $s \mapsto s^{1 / 1-\alpha}$ is locally Lipschitz continuous since $1 /(1-\alpha)>2$. If $\tau_{1}, \tau_{2}>0$ and $\tau_{2}-1 \leq \eta \leq \tau_{1}-1$ then

$$
\begin{aligned}
\rho_{\phi}\left(\psi\left(\eta, \tau_{1}\right), \psi\left(\eta, \tau_{2}\right)\right)=\left(\tau_{1}-1-\eta\right)^{1 / 2(1-\alpha)} \leq\left(\tau_{1}-\tau_{2}\right)^{1 / 2(1-\alpha)} \\
\leq C\left|\tau_{1}-\tau_{2}\right|^{1 / 2} .
\end{aligned}
$$

If $\tau_{1}>0, \tau_{2} \leq 0$ and $\eta \leq \tau_{1}-1$ we can restrict to $\eta \geq-1$ to get

$$
\begin{aligned}
\rho_{\phi}\left(\psi\left(\eta, \tau_{1}\right), \psi\left(\eta, \tau_{2}\right)\right)= & \left(\left(\tau_{1}-1-\eta\right)^{1 / 1-\alpha}-\tau_{2}\right)^{1 / 2} \\
& \leq\left(\tau_{1}^{1 / 1-\alpha}-\tau_{2}\right)^{1 / 2} \leq\left(C \tau_{1}-(C \vee 1) \tau_{2}\right)^{1 / 2} .
\end{aligned}
$$

The remaining cases $\tau_{1}>0, \tau_{2} \leq 0, \eta>\tau_{1}-1$ and $\tau_{1}, \tau_{2} \leq 0$ are easy to handle.

The problem of finding bi-Lipschitz (or even just Lipschitz) parametrizations of $\mathbb{H}$-regular surfaces in $\mathbb{H}^{n}, n \geq 2$, is still open even for smooth hypersurfaces. The model space should be $\mathbb{R} \times \mathbb{H}^{n-1} \equiv \mathbb{R}^{2 n}$ endowed with the product distance

$$
\varrho\left((\eta, A),\left(\eta^{\prime}, A^{\prime}\right)\right):=\left|\eta-\eta^{\prime}\right|+d_{\infty}\left(A, A^{\prime}\right), \quad(\eta, A),\left(\eta^{\prime}, A^{\prime}\right) \in \mathbb{R} \times \mathbb{H}^{n-1} .
$$

It can be easily seen that this distance is equivalent to the restriction of $d_{\infty}$ to $\mathbb{R}^{2 n} \equiv \mathbb{W}$, as both of them are homogeneous and left invariant on $\mathbb{W}$.

Moreover, it is not clear whether the statement of Theorem 1.1 extends to the higher dimensional case $n \geq 2$; namely, if there exist $\mathbb{H}$-regular hypersurfaces in $\mathbb{H}^{n}$ that are not bi-Lipschitz homeomorphic to $\mathbb{R} \times \mathbb{H}^{n}$. Notice, for instance, that $\mathbb{R} \times \mathbb{H}^{n-1}$ is connected by means of 
finite length curves; the same happens for any $\mathbb{H}$-regular surface, the subgroup $\mathbb{W}$ being always connected by integral curves of $\operatorname{span}\left\{X_{2}, \ldots, X_{n}\right.$, $\left.W^{\phi}, Y_{2}, \ldots, Y_{n}\right\}$ when $n \geq 2($ see $[\mathbf{2}],[\mathbf{7}])$.

\section{Proof of Theorem 1.2}

This section is devoted to the proof of Theorem 1.2, that will follow from Lemma 4.1 below in case $\Phi$ is not the inclusion $\omega \subset \mathbb{W} \hookrightarrow \mathbb{H}^{n}$ (i.e. when $\phi \not \equiv 0$ ). When $\phi \equiv 0$, Theorem 1.2 still holds when $d$ is the Euclidean distance on $\omega$ (see Remark 4.2) but not for $d=d_{c \mid \omega}$, since in this case $\Phi$ is clearly an isometry.

In the following, $d$ will be used to denote any of the metric $d_{c \mid \omega}$ or the Euclidean distance on $\omega$; we will just use the fact that, locally in $\omega$,

$$
d\left(\left(y_{1}, z, t\right),\left(y_{1}^{\prime}, z, t\right)\right) \leq C\left|y_{1}-y_{1}^{\prime}\right|
$$

for some $C>0$. Lemma 4.1 treats only the case $n \geq 2$, the generalization to $n=1$ being straightforward.

Lemma 4.1. Let $\phi: \omega \subset \mathbb{R}^{2 n} \rightarrow \mathbb{R}$ be a continuous, not identically vanishing function; then there exists no measurable function $g: \omega \rightarrow$ $[0,+\infty]$ such that

(a) $g$ is $\mathcal{L}^{2 n}$-a.e. finite;

(b) for any $A=\left(y_{1}, x_{2}, \ldots, x_{n}, y_{2}, \ldots, y_{n}, t\right), A^{\prime}=\left(y_{1}^{\prime}, x_{2}^{\prime}, \ldots, x_{n}^{\prime}\right.$, $\left.y_{2}^{\prime}, \ldots, y_{n}^{\prime}, t^{\prime}\right) \in \omega$ it holds

$$
\begin{aligned}
\left|t^{\prime}-t-\frac{1}{2}\left(\phi\left(A^{\prime}\right)+\phi(A)\right)\left(y_{1}^{\prime}-y_{1}\right)+\frac{1}{2} \sum_{j=2}^{n}\left(x_{j}^{\prime} y_{j}-x_{j} y_{j}^{\prime}\right)\right|^{1 / 2} & \\
& \leq\left(g\left(A^{\prime}\right)+g(A)\right) d\left(A^{\prime}, A\right) .
\end{aligned}
$$

Proof: We reason by contradiction. Since $\mathcal{L}^{2 n}(\{\phi \neq 0\} \cap\{|g|<+\infty\})>$ 0 there exist $\bar{z}, \bar{t}$ such that

$$
\mathcal{L}^{1}\left(\left\{y_{1} \in \mathbb{R}:\left(y_{1}, \bar{z}, \bar{t}\right) \in \omega, \phi\left(y_{1}, \bar{z}, \bar{t}\right) \neq 0,\left|g\left(y_{1}, \bar{z}, \bar{t}\right)\right|<\infty\right\}\right)>0 .
$$

In particular there is $M>0$ with $\mathcal{L}^{1}\left(E_{M}\right)>0$, where $E_{M}$ is defined by

$$
E_{M}:=\left\{y \in \mathbb{R}:\left(y_{1}, \bar{z}, \bar{t}\right) \in \omega, \phi\left(y_{1}, \bar{z}, \bar{t}\right) \neq 0,\left|g\left(y_{1}, \bar{z}, \bar{t}\right)\right| \leq M\right\} .
$$

Let us choose a Lebesgue point $\bar{y} \in E_{M}$ of $E_{M}$; in particular, $\phi\left(\bar{y}_{1}, \bar{z}, \bar{t}\right) \neq$ $0,\left|g\left(\bar{y}_{1}, \bar{z}, \bar{t}\right)\right| \leq M$ and there exists a sequence $\left\{y_{1}^{j}\right\}_{j \in \mathbb{N}} \subset E_{M}$ with $y_{1}^{j} \rightarrow$ $\bar{y}_{1}$. By exploiting condition (b) and using (4.1) for points $A=\left(\bar{y}_{1}, \bar{z}, \bar{t}\right)$, 
$A^{j}=\left(y_{1}^{j}, \bar{z}, \bar{t}\right)$ we achieve

$$
\begin{aligned}
\left|\frac{1}{2}\left(\phi(A)+\phi\left(A^{j}\right)\right)\left(y_{1}^{j}-\bar{y}_{1}\right)\right|^{1 / 2} & \leq\left(g(A)+g\left(A^{j}\right)\right) d\left(A, A^{j}\right) \\
& \leq 2 C M\left|y_{j}-\bar{y}\right|
\end{aligned}
$$

whence

$$
\left|\phi(A)+\phi\left(A^{j}\right)\right| \leq 8 C^{2} M^{2}\left|y_{1}^{j}-\bar{y}_{1}\right| .
$$

We then let $j \rightarrow \infty$ and use the continuity of $\phi$ to obtain $\phi(A)=0$, a contradiction.

Remark 4.2. When $\phi \equiv 0$ and $d$ is the Euclidean distance on $\omega$, the statement of Lemma 4.1 (and consequently Theorem 1.2) still holds. Reasoning as before, we can in fact choose $\bar{y}_{1}, \bar{z}$ and $M>0$ such that

$$
\mathcal{L}^{1}\left(\left\{t \in \mathbb{R}:\left(\bar{y}_{1}, \bar{z}, t\right) \in \omega,\left|g\left(\bar{y}_{1}, \bar{z}, t\right)\right| \leq M\right\}\right)>0 .
$$

Consider then a Lebesgue point $\bar{t}$ for the set in (4.2) and a sequence $t^{j} \rightarrow$

$\bar{t}$ in the same set. Exploiting condition (b) and using (4.1) we now obtain

$$
\left|t^{j}-\bar{t}\right|^{1 / 2} \leq 2 C M\left|t^{j}-\bar{t}\right|
$$

for any $j$, a contradiction.

\section{References}

[1] L. Ambrosio And B. Kirchheim, Rectifiable sets in metric and Banach spaces, Math. Ann. 318(3) (2000), 527-555.

[2] L. Ambrosio, F. Serra Cassano, and D. Vittone, Intrinsic regular hypersurfaces in Heisenberg groups, J. Geom. Anal. 16(2) (2006), 187-232.

[3] L. Ambrosio And P. Tilli, "Topics on analysis in metric spaces", Oxford Lecture Series in Mathematics and its Applications 25, Oxford University Press, Oxford, 2004.

[4] Z. M. BALOGH, Size of characteristic sets and functions with prescribed gradient, J. Reine Angew. Math. 564 (2003), 63-83.

[5] F. Bigolin and F. Serra Cassano, Intrinsic regular graphs in Heisenberg groups vs. weak solutions of non linear first-order PDEs, Adv. Calc. Var. (to appear).

[6] L. Capogna, D. Danielli, S. D. Pauls, and J. Tyson, "An introduction to the Heisenberg group and the sub-Riemannian isoperimetric problem", Progress in Mathematics 259, Birkhäuser Verlag, Basel, 2007.

[7] G. Citti and M. Manfredini, Implicit function theorem in Carnot-Carathéodory spaces, Commun. Contemp. Math. 8(5) (2006), 657-680. 
[8] D. R. Cole and S. D. Pauls, $C^{1}$ hypersurfaces of the Heisenberg group are $N$-rectifiable, Houston J. Math. 32(3) (2006), 713-724 (electronic).

[9] H. Federer, "Geometric measure theory", Die Grundlehren der mathematischen Wissenschaften 153, Springer-Verlag New York Inc., New York, 1969.

[10] B. Franchi, R. Serapioni, and F. Serra Cassano, Rectifiability and perimeter in the Heisenberg group, Math. Ann. 321(3) (2001), 479-531.

[11] B. Franchi, R. Serapioni, and F. Serra Cassano, Regular submanifolds, graphs and area formula in Heisenberg groups, Adv. Math. 211(1) (2007), 152-203.

[12] B. Kirchheim and F. Serra Cassano, Rectifiability and parameterization of intrinsic regular surfaces in the Heisenberg group, Ann. Sc. Norm. Super. Pisa Cl. Sci. (5) 3(4) (2004), 871-896.

[13] P. Mattila, R. Serapioni, and F. Serra Cassano, Characterizations of intrinsic rectifiability in Heisenberg groups, Ann. Sc. Norm. Super. Pisa Cl. Sci. (5) (to appear).

[14] S. D. PAuls, A notion of rectifiability modeled on Carnot groups, Indiana Univ. Math. J. 53(1) (2004), 49-81.

[15] D. Vittone, Submanifolds in Carnot groups, Tesi di Perfezionamento, Scuola Normale Superiore, Pisa (2008).

Francesco Bigolin:

Dipartimento di Matematica

Università degli Studi di Trento

Via Sommarive 14

38050 Povo (TN)

Italy

E-mail address: bigolin@science.unitn.it

Davide Vittone:

Dipartimento di Matematica Pura ed Applicata

Università degli Studi di Padova

Via Trieste 63

35121 Padova (PD)

Italy

E-mail address: vittone@math.unipd.it

Primera versió rebuda el 8 d'octubre de 2008, darrera versió rebuda el 21 de gener de 2009. 